\title{
Saúde mental e qualidade de vida dos estudantes de faculdades de medicina brasileiras: uma revisão integrativa
}

\author{
Mental health and quality of life of students from Brazilian medical schools: a literature integrative \\ Salud mental y calidad de vida de estudiantes de las facultades de medicina brasileñas: una \\ revisión integradora
}

Giulia Machado Caldeira Ardisson ${ }^{1 *}$, Rafaela de Oliveira Andrade ${ }^{1}$, Alexandre Vazzoler Andrião Aline Cristina Mafra ${ }^{1}$, Maria Carmela Kneip Lopes Fonseca ${ }^{1}$, Mateus Gonçalves Amâncio', Tauane Larissa Menão ${ }^{1}$, Maria José Guedes Gondim Almeida'.

\section{RESUMO}

Objetivo: Discutir a saúde mental dos estudantes de medicina brasileiros, identificar fatores de risco e o papel das escolas médicas. Métodos: A pesquisa foi realizada por meio de descritores associados à saúde mental dos estudantes de medicina nas plataformas PubMed, LILACS e Scielo. O acesso ocorreu entre outubro e dezembro de 2020. Foram selecionados 53 artigos dentro dos seguintes critérios: artigos publicados a partir de 2014, transversais ou longitudinais, que avaliaram estudantes de medicina brasileiros quanto à saúde mental. Desses, foram excluídos 34 artigos por não se relacionarem ao tema central. Resultados: Estudantes de medicina exibem pior bem-estar psicossocial do que outros jovens. Existe alta prevalência de desordens como depressão, ansiedade, estresse, síndrome de burnout e abuso de substâncias. Dentre os fatores de risco, mulheres são um grupo de vulnerabilidade. Em relação a morar sozinho, os resultados encontrados foram divergentes. Ademais, há relação entre grade curricular do curso e saúde mental e qualidade de vida. A respeito do método de aprendizagem, há preferência dos estudantes pelo ensino ativo. Considerações finais: Diversos fatores envolvidos na formação médica influenciam diretamente na saúde mental e qualidade de vida dos acadêmicos. Portanto, é importante estabelecer medidas e mecanismos de suporte para esse grupo.

Palavras chave: Estudantes de medicina, Saúde mental, Qualidade de vida, Brasil.

\begin{abstract}
Objective: To discuss the mental health of Brazilian medical students, to identify risk factors and the role of medical schools. Methods: The research was carried out through the platforms PubMed, LILACS and Scielo, using descriptors associated with the mental health of medical students. Access occurred between October and December 2020. Fifty three articles were selected according to the following criteria: articles published from 2014, transversal or longitudinal, which evaluated Brazilian medical students regarding mental health. Among these, 34 articles were excluded because they were not directly linked to the central theme. Results: Medical students show worse psychosocial well-being than other young people in general. There is a high prevalence of disorders such as depression, anxiety, stress, burnout syndrome and substance abuse. Among the risk factors, women are considered a vulnerable group. Regarding living alone, the results found were divergent. Furthermore, there is a relationship between the course curriculum and mental health and quality of life. Regarding the learning method, students prefer active learning. Final considerations: Several factors involved in medical education directly influence the mental health and quality of life of academics. Therefore, it is important to establish support measures and mechanisms for this group.
\end{abstract}

Keywords: Students medical, Mental health, Quality of life, Brazil.

\section{RESUMEN}

Objetivo: Discutir la salud mental de estudiantes de medicina brasileños, identificar factores de riesgo y el papel de las escuelas de medicina. Métodos: La investigación fue realizada a través de PubMed, LILACS y Scielo utilizando descriptores sobre salud mental de estudiantes de medicina. El acceso ocurrió entre octubre y diciembre de 2020. Fueron seleccionados 53 artículos dentro de los criterios: publicados a partir de 2014, transversales o longitudinales, evaluaron estudiantes de medicina brasileños acerca de salud mental. De

1 Universidade Federal de Juiz de Fora (UFJF), Juiz de Fora - MG. *E-mail: ardissongiulia@gmail.com 
esos, 34 artículos fueron excluidos porque se desviaron del tema central. Resultados: Estudiantes de medicina tienen peor bienestar psicosocial que otros jóvenes. Hay una alta prevalencia de trastornos como depresión, ansiedad, estrés, síndrome de Burnout y abuso de sustancias. Entre los factores de riesgo, mujeres son consideradas un grupo de vulnerabilidad. Respecto a vivir solo, los resultados encontrados fueron divergentes. Además, hay relación entre el currículo del curso y salud mental y calidad de vida. Respecto al método de aprendizaje, los estudiantes prefieren la enseñanza activa. Consideraciones finales: Varios factores involucrados en la formación médica influyen directamente en la salud mental y calidad de vida de los estudiantes. Por lo tanto, es importante establecer medidas y mecanismos de apoyo a ese grupo.

Palabras clave: Estudiantes de medicina, Salud mental, Calidad de vida, Brasil.

\section{INTRODUÇÃO}

As escolas médicas são mundialmente reconhecidas por serem ambientes estressantes para o aluno. A literatura relata que estudantes de medicina apresentam pior bem-estar psicossocial quando comparados a colegas da mesma idade, e maior prevalência de transtornos mentais como ansiedade, depressão e burnout do que a população em geral (PACHECO JP, et al., 2017).

Fatores como a intensa carga de estudos, sobrecarga de informações, pouco tempo livre, restrições financeiras, mais de 15 horas de atividades extracurriculares e competição por alto desempenho são apontados em alguns estudos como possíveis causas de adoecimento nessa população (MOUTINHO ILD, 2018).

Um estudo realizado nos Estados Unidos avaliou e comparou a saúde mental e o uso de drogas em acadêmicos de medicina dos $1^{\circ}, 2^{\circ}$ e $3^{\circ}$ anos com estudantes de outros cursos da Universidade de Vanderbilt. Os resultados mostraram altos níveis de ansiedade entre os estudantes. Os dados demonstraram escores significativamente maiores para as mulheres (GHODASARA SL, et al., 2011).

Uma metanálise, que englobou estudos transversais utilizando instrumentos validados, analisou dados de 62.728 estudantes de medicina do Oriente Médio, da Europa, da América do Norte, da América do Sul e da Ásia. Os resultados apontaram para uma prevalência geral de depressão de $28,0 \%$ (PUTHRAN R, et al., 2016).

Estudos brasileiros também apresentam dados alarmantes. Pesquisadores de Minas Gerais verificaram a frequência e as características dos sintomas depressivos em estudantes de medicina. Desses, 29.9\% apresentavam sintomas de depressão e 30,1\% sintomas de ansiedade (MOUTINHO ILD, 2018). Uma revisão sistemática e metanálise de 59 trabalhos sobre a saúde mental dos acadêmicos das escolas médicas brasileiras demonstrou prevalência de ansiedade de 32,9\%, impactando na qualidade de vida e nos níveis de empatia dos estudantes (PACHECO JP, et al., 2017).

A ocorrência de distúrbios de humor e ansiedade na graduação, quando não adequadamente tratados, podem se perpetuar ou agravar durante a atividade profissional. A ampliação do conhecimento da realidade brasileira acerca do tema é de extrema relevância no contexto atual (VASCONCELOS T, 2015).

Dessa forma, este estudo tem por objetivo apresentar um panorama da saúde mental e qualidade de vida dos estudantes de medicina no Brasil; identificar os principais fatores de risco envolvidos; e abordar o papel das escolas médicas nesse contexto.

\section{MÉTODOS}

Esta revisão de literatura fez uso dos pesquisadores eletrônicos PubMed, Literatura Latino-Americano e do Caribe (LILACS), Science Direct e Biblioteca Científica Eletrônica Online (Scielo). A pesquisa foi feita com três buscas utilizando descritores de assunto em saúde (DECS/MESH): ((Quality of life) OR (Burnout syndrome) OR (Mental health) OR (Psychological stress) OR (Anxiety) OR (Lifestyle) OR (Depression)) AND (medical students AND Brazil); ((Qualidade de vida) OR (Saúde mental) OR (Estresse Psicológico) OR (Depressão) OR (Ansiedade) OR (estilo de vida)) AND (estudantes de medicina); e ((Education, Medical, Undergraduate) AND (problem-based learning)) AND (anxiety). 
O acesso às bases de dados ocorreu entre os meses de outubro e dezembro de 2020. Dos artigos elegíveis, ou seja, disponíveis gratuitamente completos; em Português e Inglês; e com data de publicação entre o ano de 2014 até outubro de 2020, apenas cinquenta e três artigos foram selecionados por estarem de acordo com os critérios de inclusão. Assim, incluímos estudos que: 1) foram feitos a partir de 2014 ;2) fossem transversais ou longitudinais; 3) avaliaram alunos de medicina em atividade nas faculdades de medicina brasileiras; 4) relacionaram aspectos da saúde mental.

A fim de sistematizar as informações de interesse dos artigos incluídos, os dados foram organizados em diferentes grandes temas: "Diferença entre homens e mulheres"; "Alunos que moram com os pais ou não"; "Influência do currículo acadêmico na saúde mental do estudante de medicina"; e "Aprendizagem baseada em problemas e saúde mental".

Todos os artigos selecionados foram lidos integralmente, e posteriormente, passaram por processo de seleção, no qual, 34 artigos foram excluídos por: 1) Fugir do tema central; 2) Não contemplar os grandes eixos temáticos propostos pela nossa equipe.

Figura 1 - Fluxograma de metodologia.

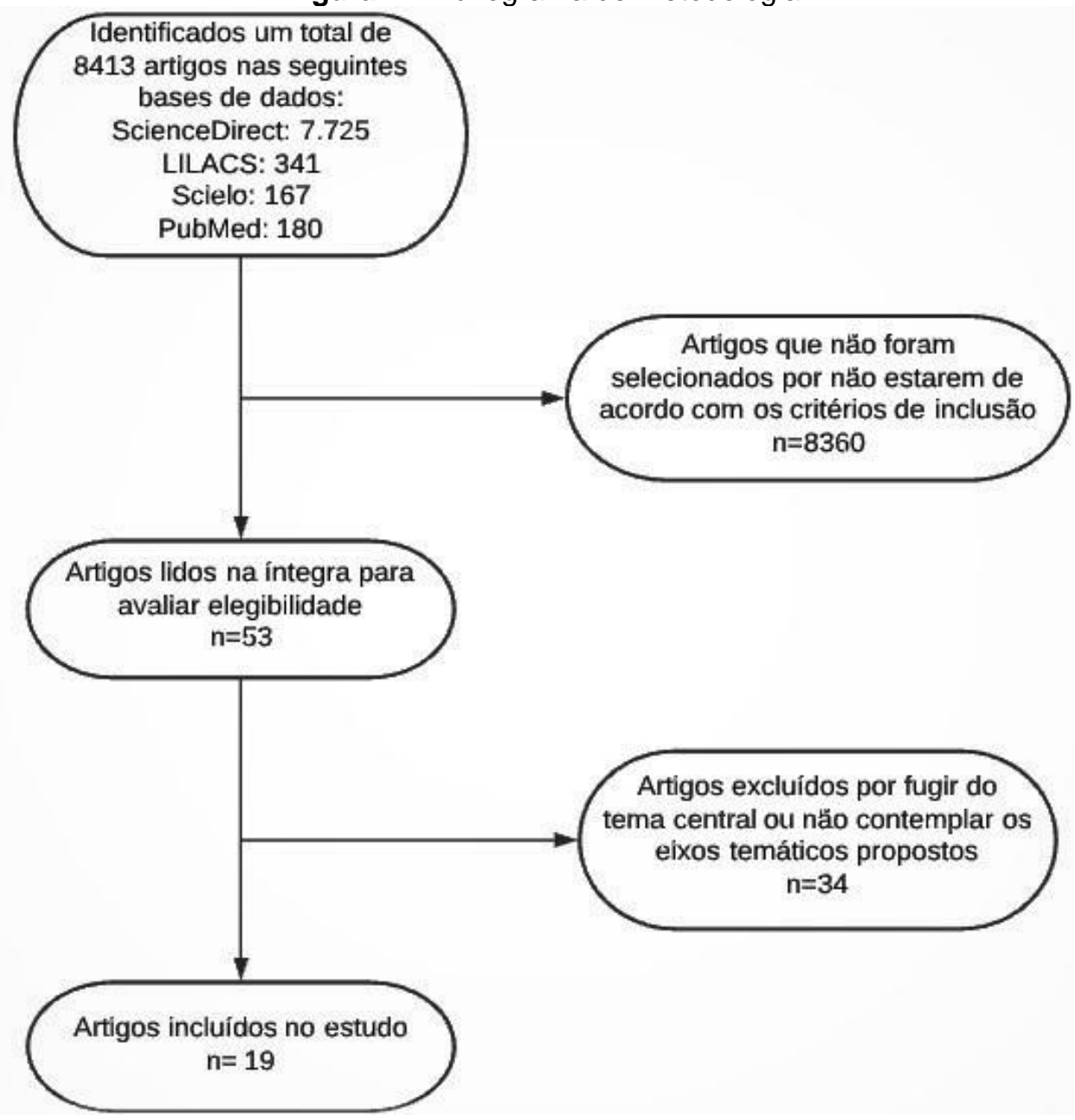

Fonte: Ardisson GMC, et al., 2021.

\section{RESULTADOS E DISCUSSÃO}

Nesta revisão de literatura abordaremos 4 fatores que estão associados à piora da qualidade de vida dos estudantes de medicina. Foi comparada à saúde mental desse grupo com a de outros estudantes universitários; a prevalência de ansiedade e depressão entre os gêneros; a associação com questões habitacionais; a influência do currículo acadêmico no contexto de estresse, incluindo a relação com o método de estudo baseado em problemas, que é utilizado em algumas universidades (Quadro 1). 
Quadro 1 - Tabela com os principais achados dos artigos selecionados para a revisão integrativa da literatura.

\begin{tabular}{|c|c|c|c|c|}
\hline Título & $\begin{array}{c}\text { Ano de } \\
\text { Publicação }\end{array}$ & Autores & Revista & Principais Achados \\
\hline $\begin{array}{l}\text { Saúde mental e qualidade de vida de } \\
\text { estudantes de medicina brasileiros: } \\
\text { incidência, prevalência e fatores } \\
\text { associados em dois anos de seguimento. }\end{array}$ & 2019 & $\begin{array}{l}\text { MOUTINHO } \\
\text { ILD, et al. }\end{array}$ & $\begin{array}{l}\text { Psychiatry } \\
\text { Reserarch }\end{array}$ & $\begin{array}{l}\text { Alta prevalência de desordens emocionais entre estudantes de } \\
\text { medicina. Dos estudantes acompanhados, } 30 \% \text { apresentaram } \\
\text { sintomas depressivos e } 13 \% \text { tiveram sobreposição de } \\
\text { depressão, ansiedade e estresse. Há prejuízo na qualidade de } \\
\text { vida das mulheres em comparação aos homens. }\end{array}$ \\
\hline $\begin{array}{l}\text { Prevalência de Transtornos Mentais } \\
\text { Comuns e Fatores Associados em } \\
\text { Estudantes de Medicina: um Estudo } \\
\text { Comparativo. }\end{array}$ & 2016 & $\begin{array}{l}\text { FERREIRA } \\
\text { CMG, et al. }\end{array}$ & $\begin{array}{l}\text { Revista Brasileira } \\
\text { de Educação } \\
\text { Médica. }\end{array}$ & $\begin{array}{l}\text { Metade dos estudantes apresentam altos níveis de depressão, } \\
\text { ansiedade e estresse. A saúde mental do estudante apresenta } \\
\text { diferenças consideráveis no início e no fim do semestre. }\end{array}$ \\
\hline $\begin{array}{l}\text { Relação entre Fatores Acadêmicos e a } \\
\text { Saúde Mental de Estudantes } \\
\text { Universitários. }\end{array}$ & 2018 & $\begin{array}{l}\text { ARINO DO, } \\
\text { BARDAGI MP. }\end{array}$ & $\begin{array}{l}\text { Psicologia em } \\
\text { pesquisa }\end{array}$ & $\begin{array}{l}\text { Em universitários em geral, é prevista a prevalência de } 15 \text { a } 25 \% \\
\text { de transtornos mentais. }\end{array}$ \\
\hline $\begin{array}{l}\text { Fatores associados à depressão e } \\
\text { ansiedade em estudantes de medicina: um } \\
\text { estudo multicêntrico. }\end{array}$ & 2016 & $\begin{array}{l}\text { MAYER FB, et } \\
\text { al. }\end{array}$ & $\begin{array}{l}\text { BMC Medical } \\
\text { Education }\end{array}$ & $\begin{array}{l}\text { A prevalência de sintomas depressivos é } 41,3 \% \text { em estudantes } \\
\text { de medicina brasileiros, maior que a global, que é } 28 \% \text {. Não } \\
\text { confirmou a hipótese de que alunos que moram sozinhos } \\
\text { possuem piores escores de ansiedade e depressão. }\end{array}$ \\
\hline $\begin{array}{l}\text { Prevalência e Fatores Associados à } \\
\text { Depressão e Ansiedade entre Estudantes } \\
\text { Universitários da Área da Saúde de um } \\
\text { Grande Centro Urbano do Nordeste do } \\
\text { Brasil. }\end{array}$ & 2018 & LEAO AM, et al. & $\begin{array}{l}\text { Revista Brasileira } \\
\text { de Educação } \\
\text { Médica }\end{array}$ & $\begin{array}{l}\text { O curso de medicina ocupou o quarto lugar em níveis de } \\
\text { depressão e o quinto em ansiedade quando comparado aos } \\
\text { outros cursos da saúde. Um dos aspectos mais fortemente } \\
\text { associados à ansiedade foi a baixa quantidade e qualidade de } \\
\text { sono. }\end{array}$ \\
\hline $\begin{array}{l}\text { Síndrome de burnout, atividades } \\
\text { extracurriculares e apoio social entre } \\
\text { estudantes de medicina brasileiros: uma } \\
\text { análise transversal. }\end{array}$ & 2020 & $\begin{array}{l}\text { FONTANA } \\
\text { MCP, et al. }\end{array}$ & $\begin{array}{l}\text { BMC medical } \\
\text { education }\end{array}$ & $\begin{array}{l}\text { Altos valores de Síndrome de Burnout entre os estudantes de } \\
\text { medicina }\end{array}$ \\
\hline
\end{tabular}




\begin{tabular}{|c|c|c|c|c|}
\hline Título & $\begin{array}{c}\text { Ano de } \\
\text { Publicação }\end{array}$ & Autores & Revista & Principais Achados \\
\hline $\begin{array}{l}\text { Estressores, sofrimento psíquico e } \\
\text { problemas de saúde mental entre } \\
\text { estudantes de medicina brasileiros. }\end{array}$ & 2019 & $\begin{array}{l}\text { CASTALDELLI- } \\
\text { MAIA JM, et al. }\end{array}$ & $\begin{array}{l}\text { International } \\
\text { Review of } \\
\text { Psychiatry }\end{array}$ & A prevalência de problemas com álcool chega a 32,9\%. \\
\hline $\begin{array}{l}\text { Saúde Mental do Estudante de Medicina: } \\
\text { Psicopatologia, Estresse, Sono e } \\
\text { Qualidade de Vida. }\end{array}$ & 2018 & $\begin{array}{l}\text { AMORIM BB, et } \\
\text { al. }\end{array}$ & $\begin{array}{l}\text { Revista Psicologia, } \\
\text { Diversidade e } \\
\text { Saúde. }\end{array}$ & $\begin{array}{l}\text { Bebida pode causar danos pessoais, prejuízo no } \\
\text { desenvolvimento e na estruturação de habilidades cognitivo- } \\
\text { comportamentais e emocionais }\end{array}$ \\
\hline $\begin{array}{l}\text { Dilemas e frustrações do estudante de } \\
\text { medicina: estudo transversal. }\end{array}$ & 2018 & FEIJÓ DH, et al. & $\begin{array}{l}\text { Pará Research } \\
\text { Medical Journal }\end{array}$ & $\begin{array}{l}\text { O abuso de álcool e de tabaco são práticas perpetuadas na } \\
\text { profissão médica. O ciclo básico, devido ao pouco contato com } \\
\text { pacientes, representa uma quebra das expectativas dos } \\
\text { estudantes. O terceiro e quarto anos de graduação podem } \\
\text { piorar a autoestima do aluno. }\end{array}$ \\
\hline $\begin{array}{l}\text { Sintomas de depressão em universitários } \\
\text { de medicina. }\end{array}$ & 2019 & $\begin{array}{l}\text { AQUINO DR, et } \\
\text { al. }\end{array}$ & $\begin{array}{l}\text { Boletim Academia } \\
\text { Paulista de } \\
\text { Psicologia }\end{array}$ & $\begin{array}{l}\text { A faculdade de medicina tem impacto negativo sobre a saúde } \\
\text { mental dos alunos. O período inicial da faculdade foi apontado } \\
\text { como mais difícil. Porém, } 90,1 \% \text { dos estudantes não possuíam } \\
\text { disciplinas pendentes e } 95,9 \% \text { não necessitaram repetir algum } \\
\text { período. No entanto, evidenciou-se a presença de diversos } \\
\text { sintomas depressivos entre eles. Além disso,a presença de } \\
\text { sono irregular dificulta os processos mentais de pensamento e } \\
\text { julgamento.A dependência financeira de terceiros por um longo } \\
\text { período, especialmente entre os alunos de baixa renda, pode } \\
\text { gerar perda da qualidade de vida e quadros de angústia e } \\
\text { depressão. }\end{array}$ \\
\hline $\begin{array}{l}\text { Privação do sono e sonolência excessiva } \\
\text { em médicos residentes e estudantes de } \\
\text { medicina. }\end{array}$ & 2016 & $\begin{array}{l}\text { PURIM KSM, et } \\
\text { al. }\end{array}$ & $\begin{array}{l}\text { Revista do Colégio } \\
\text { Brasileiro de } \\
\text { Cirurgiões }\end{array}$ & $\begin{array}{l}\text { A insatisfação com quantidade de sono, insônia e inatividade } \\
\text { física foram aspectos fortemente associados à saúde mental. } \\
\text { Muitos estudantes de medicina têm perdido a qualidade do } \\
\text { sono. }\end{array}$ \\
\hline
\end{tabular}




\begin{tabular}{|c|c|c|c|c|}
\hline Título & $\begin{array}{c}\text { Ano de } \\
\text { Publicação }\end{array}$ & Autores & Revista & Principais Achados \\
\hline $\begin{array}{l}\text { Comparação da qualidade de vida entre } \\
\text { estudantes de medicina e população } \\
\text { jovem em geral. }\end{array}$ & 2015 & $\begin{array}{l}\text { PAGNIN D, DE } \\
\text { QUEIROZ V }\end{array}$ & $\begin{array}{l}\text { Education for } \\
\text { health (Abingdon, } \\
\text { England). }\end{array}$ & $\begin{array}{l}\text { Existe pior bem-estar psicológico e piores relações sociais do } \\
\text { que jovens da mesma idade }\end{array}$ \\
\hline $\begin{array}{l}\text { Percepção da qualidade de vida e fatores } \\
\text { associados aos escores de qualidade de } \\
\text { vida de alunos de uma escola de medicina. }\end{array}$ & 2017 & $\begin{array}{l}\text { CUNHA DHF, et } \\
\quad \text { al. }\end{array}$ & $\begin{array}{l}\text { Jornal Brasileiro de } \\
\text { Psiquiatria }\end{array}$ & $\begin{array}{l}\text { Mulheres são consideradas um grupo de vulnerabilidade no que } \\
\text { se refere à saúde mental. Os estudantes que residem sozinhos } \\
\text { apresentam menores escores no domínio físico. }\end{array}$ \\
\hline $\begin{array}{l}\text { Prevalência e Fatores Associados à } \\
\text { Depressão e Ansiedade entre Estudantes } \\
\text { Universitários da Área da Saúde de um } \\
\text { Grande Centro Urbano do Nordeste do } \\
\text { Brasil. }\end{array}$ & 2018 & LEAO AM, et al. & $\begin{array}{l}\text { Revista Brasileira } \\
\text { de Educação } \\
\text { Médica. }\end{array}$ & $\begin{array}{l}\text { Não encontrou associação significativa entre morar com } \\
\text { pais/cônjuge ou outra situação habitacional com o fato de ter } \\
\text { depressão. }\end{array}$ \\
\hline $\begin{array}{l}\text { Saúde mental de acadêmicos do primeiro } \\
\text { ano da faculdade de medicina: uma } \\
\text { abordagem de gênero. }\end{array}$ & 2018 & $\begin{array}{l}\text { MEDEIROS } \\
\text { MRB, et al. }\end{array}$ & $\begin{array}{l}\text { Revista Brasileira } \\
\text { de Educação } \\
\text { Médica }\end{array}$ & $\begin{array}{l}\text { A prevalência de sintomas de estresse, depressivos, de } \\
\text { sonolência diurna e esgotamento profissional em estudantes } \\
\text { ingressantes no curso médico foi bastante significativa. Houve } \\
\text { diferença no componente mental da qualidade de vida e na } \\
\text { presença de Transtornos Mentais Comuns em relação ao sexo, } \\
\text { sendo que as mulheres apresentaram os piores resultados. }\end{array}$ \\
\hline $\begin{array}{l}\text { Qualidade de Vida de Estudantes de } \\
\text { Medicina em um Curso que Adota } \\
\text { Metodologias Ativas de Ensino- } \\
\text { Aprendizagem. }\end{array}$ & 2018 & $\begin{array}{l}\text { CHAGAS NB, et } \\
\quad \text { al. }\end{array}$ & $\begin{array}{l}\text { Revista Brasileira } \\
\text { de Educação } \\
\text { Médica. }\end{array}$ & $\begin{array}{l}\text { Há estreita relação entre a grade curricular do curso de medicina } \\
\text { e a saúde mental e qualidade de vida do estudante de medicina. } \\
\text { O terceiro ano da graduação médica foi destacado como o de } \\
\text { pior escore de qualidade de vida. }\end{array}$ \\
\hline $\begin{array}{l}\text { Mecanismos de defesa e qualidade de vida } \\
\text { de estudantes de medicina de acordo com } \\
\text { a fase de graduação. }\end{array}$ & 2020 & $\begin{array}{l}\text { BORGES GBM, } \\
\quad \text { et al. }\end{array}$ & $\begin{array}{l}\text { Trends Psychiatry } \\
\text { Psychother. }\end{array}$ & $\begin{array}{l}\text { Fases iniciais do curso médico, quando comparados àqueles } \\
\text { nas fases finais, apresentaram significativamente mais sintomas } \\
\text { depressivos, pior qualidade de vida e maiores escores na } \\
\text { avaliação de neurose. }\end{array}$ \\
\hline
\end{tabular}




\begin{tabular}{|c|c|c|c|c|}
\hline Título & $\begin{array}{c}\text { Ano de } \\
\text { Publicação }\end{array}$ & Autores & Revista & Principais Achados \\
\hline $\begin{array}{l}\text { Aprendizagem baseada em problemas em } \\
\text { comparação com a aprendizagem } \\
\text { baseada em palestras entre estudantes de } \\
\text { medicina. }\end{array}$ & 2016 & $\begin{array}{l}\text { RIZWAN F, et } \\
\text { al. }\end{array}$ & $\begin{array}{c}\text { Journal Of Pakistan } \\
\text { Medical } \\
\text { Association }\end{array}$ & $\begin{array}{l}\text { Alunos que foram direcionados à coorte da metodologia não } \\
\text { tradicional obtiveram melhor resultados. }\end{array}$ \\
\hline $\begin{array}{l}\text { Envolvendo pesquisadores novatos no } \\
\text { processo e na cultura da ciência usando } \\
\text { uma estratégia de caso "Passe o } \\
\text { problema" }\end{array}$ & 2015 & $\begin{array}{l}\text { WHITE HB, } \\
\text { USHER DC. }\end{array}$ & $\begin{array}{l}\text { Biochemistry and } \\
\text { Molecular Biology } \\
\text { Education }\end{array}$ & $\begin{array}{l}\text { A metodologia baseada em problemas tem sido considerada } \\
\text { positiva na interação social dos estudantes com os colegas. }\end{array}$ \\
\hline $\begin{array}{l}\text { Como os alunos de graduação lidam com } \\
\text { a ansiedade resultante de práticas ativas } \\
\text { de aprendizagem na introdução à } \\
\text { biologia? }\end{array}$ & 2020 & $\begin{array}{l}\text { BRIGATI JR, et } \\
\quad \text { al. }\end{array}$ & Plos One & $\begin{array}{l}\text { A necessidade de participação do estudante na aprendizagem } \\
\text { baseada em problemas pode causar insegurança. }\end{array}$ \\
\hline
\end{tabular}

Fonte: Ardisson GMC, et al., 2021. 


\section{Comparativo da Saúde mental dos estudantes de medicina}

Os estudantes de medicina estão expostos a diversos estressores ao longo do curso. E quando se analisa a saúde mental destes, nota-se que existe uma alta prevalência de desordens emocionais (MOUTINHO ILD, et al., 2019). Além disso, quase metade desses estudantes apresentam altos níveis de depressão, ansiedade e estresse (FERREIRA CMG, et al., 2016). Entretanto, para os universitários em geral, é prevista uma prevalência de 15 a 25\% em relação a transtornos mentais durante a graduação (ARIÑO DO e BARDAGI MP, 2018). Portanto, a prevalência de tais transtornos é maior entre os acadêmicos de medicina comparativamente a outros universitários, e merece ser considerada como um importante problema para a comunidade médica e acadêmica.

Em um acompanhamento de 2 anos, cerca de $30 \%$ dos estudantes acompanhados apresentaram sintomas depressivos. É importante ressaltar que, quase 13\% desses graduandos tiveram uma sobreposição de depressão, ansiedade e estresse. Assim, nota-se que um número substancial de alunos tem ansiedade e sintomas depressivos coexistentes (MOUTINHO ILD, et al., 2019).

Um estudo multicêntrico para avaliar os fatores pessoais e institucionais relacionados à prevalência de depressão e ansiedade de alunos de 22 escolas médicas brasileiras, encontrou uma prevalência de 41,3\% de sintomas depressivos em estudantes de medicina brasileiros. Essa porcentagem é maior do que a prevalência global, de $28 \%$. Isso reforça ainda mais a necessidade de atenção que este grupo de estudantes necessita (MAYER FB, et al., 2016).

Chama atenção o fato de o curso de Medicina ter ocupado o quarto lugar em níveis de depressão e o quinto em ansiedade quando comparado aos outros cursos da saúde por um estudo entre universitários da área da saúde de um grande centro urbano do nordeste do Brasil. Porém, tal fato não desmerece a atenção que deve ser dada a essa problemática nas faculdades de medicina. Na verdade, demonstra que outros cursos da área da saúde também necessitam de atenção quanto à saúde mental de seus estudantes (LEÃO AM, et al., 2018).

Ademais, alguns estudos apontaram para altos valores de Síndrome de Burnout entre os estudantes de medicina (FONTANA MCP, et al., 2020). Em uma pesquisa comparando estudantes de medicina, farmáciabioquímica e enfermagem, a prevalência da síndrome estava entre 2,5 e $26 \%$, sendo que as maiores foram entre os acadêmicos de medicina. Isso ocorre devido, principalmente, à integralidade e extensão do curso, à quantidade de matérias e às atividades extracurriculares (LEÃO AM, et al., 2018).

Além dos transtornos mentais citados, devemos considerar também o uso de substâncias. Problemas com álcool chegam a 32,9\% (CASTADELLI-MAIA JM, et al., 2019). O excessivo consumo de bebidas alcoólicas entre estudantes de Medicina é preocupante, não só pelos danos pessoais que pode causar, como pelo prejuízo no desenvolvimento e na estruturação de habilidades cognitivo-comportamentais e emocionais (AMORIM BB, et al., 2018). O abuso de álcool e de tabaco tendem a ser práticas perpetuadas na profissão médica e, em até 12\%, evoluem para a dependência (FEIJÓ DH, et al., 2018).

A formação médica parece ter um impacto negativo sobre a saúde mental dos alunos, podendo haver vários determinantes, como a carga horária exigida de estudos diários e a mudança no método de estudo (AQUINO DR, et al., 2019). Ademais, insatisfação com quantidade de sono, insônia e inatividade física foram aspectos fortemente associados à prevalência em conjunto com fatores individuais e sócio organizacionais de trabalho e estudo (PURIM KSM, et al., 2016).

Portanto, esses estudantes exibem pior bem-estar psicológico e piores relações sociais do que jovens da mesma idade. É importante ressaltar que os acadêmicos de medicina tendem a perceber que a dedicação e o tempo para o bem-estar pessoal são menos importantes do que compromissos associados aos estudos. Ademais, muitas escolas médicas não fornecem apoio social e cuidados de saúde eficazes aos alunos (PAGNIN DQV, 2015).

\section{Diferença entre homens e mulheres}

Com relação ao gênero dos estudantes de medicina, pesquisas evidenciam diferenças na qualidade de vida. As mulheres são consideradas um grupo de vulnerabilidade no que se refere à saúde mental, 
especialmente nos quesitos qualidade de vida e presença de transtornos mentais comuns. No estudo de Cunha DHF (2018) foi aplicado um questionário com estudantes de medicina do primeiro ao sexto ano da Universidade Federal de São Paulo, que também demonstrou diferenças na saúde mental entre os sexos, uma vez que o sexo feminino apresentou escores menores nos domínios físico, psicológico, nível de independência e qualidade de vida em relação ao sexo masculino.

O trabalho Moutinho ILD, et al. (2019) realizou um acompanhamento durante dois anos com estudantes de medicina da Universidade Federal de Juiz de Fora com o objetivo de avaliar a incidência, prevalência e fatores associados à qualidade de vida e aos sintomas de depressão, ansiedade e estresse de estudantes da área. Através deste estudo foi observado prejuízo na qualidade de vida das mulheres em comparação aos homens, sendo o sexo feminino considerado um fator de risco importante associado a uma baixa qualidade de vida entre os estudantes de medicina.

\section{Alunos que moram com os pais ou não}

A avaliação da qualidade de vida e a saúde mental dos estudantes de medicina abrange a questão habitacional de morar com familiares, cônjuge, amigos ou sozinho. Segundo o estudo transversal de Cunha DHF (2018), que incluiu alunos de medicina da 1aa $6^{\text {a }}$ série na EPM-Unifesp em 2011, os estudantes que residem sozinhos em relação aos que moram com o cônjuge, pais, irmãos, parentes ou amigos, apresentam menores escores no domínio físico, possivelmente em razão de possuir responsabilidades relacionadas à moradia que outros estudantes não possuem.

Em contrapartida, outras pesquisas apresentaram conclusões diferentes, como o estudo de Leão AM, et al. (2018), que avaliou aspectos relacionados à presença de depressão e ansiedade em estudantes universitários da área da saúde não encontrou associação significativa entre morar com pais/cônjuge ou outra situação habitacional com o fato de ter depressão. Além disso, o trabalho de Mayer FB, et al. (2016), analisou fatores pessoais e institucionais em associação à presença de depressão e ansiedade e também não confirmou a hipótese de que alunos que moram sozinhos possuem escores de ansiedade e depressão maiores.

Dessa forma, evidencia-se a necessidade de estudos direcionados para a questão habitacional dos estudantes de medicina para uma análise mais precisa da situação. Assim, será possível analisar mais detalhadamente a presença ou não de fatores de risco e proteção quanto à moradia dos alunos (MAYER FB, et al., 2016).

\section{Influência do currículo acadêmico na saúde mental do estudante de medicina}

Diversas pesquisas chegaram à conclusão de que há estreita relação entre a grade curricular do curso de medicina e a saúde mental e qualidade de vida do estudante de medicina. Por ser um curso de extensa carga horária, os estudantes são submetidos a diversos gatilhos que prejudicam sua saúde mental, como grande quantidade de conteúdo programático, dificuldade das avaliações e numerosas atividades extracurriculares. Além disso, a privação do sono, a falta de tempo para cuidado pessoal e interação social e o contato constante com pessoas doentes e em sofrimento são fatores presentes no dia a dia do estudante que impactam diretamente sua qualidade de vida (CHAGAS NB, et al., 2018).

No estudo de Aquino DR, et al. (2019), que avaliou sintomas de depressão em estudantes de medicina, o período inicial da faculdade foi apontado como mais difícil por mais da metade dos pesquisados $(54,5 \%)$ e, entre eles, ao menos uma única vez houve pensamentos de desistência da graduação (36,4\%). Tal resultado foi atribuído, entre outros fatores, ao fato de que o estudante já inicia o curso sob pressão e expectativas tanto pessoais quanto de familiares e amigos, além de estarem chegando, em sua maioria, com horas de estudos pré-vestibulares acumuladas. Assim, o contato com um conteúdo desconhecido e extenso, mudanças nos métodos de estudo e a grande carga horária fez com que grande parte dos estudantes relatasse o período inicial como o mais difícil.

Ainda neste estudo, 90,1\% dos estudantes não possuíam disciplinas pendentes e 95,9\% não necessitam repetir algum período, o que indica que possuíam um bom desempenho acadêmico. No entanto, evidenciou- 
se a presença de diversos sintomas depressivos entre eles, indicando que o bom desempenho pode estar relacionado com perda da qualidade de vida e saúde mental (AQUINO DR, et al., 2019).

O trabalho de Borges GBM, et al. (2020) acerca de mecanismos de defesa e qualidade de vida de estudantes de medicina de acordo com a fase de graduação corrobora esses resultados, indicando que os estudantes nas fases iniciais do curso médico, quando comparados àqueles nas fases finais, apresentaram significativamente mais sintomas depressivos, pior qualidade de vida e maiores escores na avaliação de neurose. Como uma das possíveis explicações para isso, os resultados mostraram que os alunos com estrutura curricular com maior percentual de horas com atividades clínicas relataram menor estresse. Ou seja, o pouco contato com a prática médica nas fases iniciais da graduação poderia ser um fator colaborador para o aumento da ansiedade.

O trabalho de Feijó DH, et al. (2018) sobre dilemas e frustrações do estudante de medicina aponta que durante o ciclo básico, devido ao pouco contato com pacientes, o estudo das matérias básicas representa uma quebra das expectativas dos calouros, que esperam encontrar o exibido em filmes ou livros.

Em contrapartida, em uma pesquisa o terceiro ano da graduação médica foi destacado como o de pior escore de qualidade de vida (CHAGAS MB, et al., 2018). De acordo com esse estudo, algo que poderia explicar esse declínio na qualidade de vida é a transição do ciclo básico para a fase clínica do curso, com o primeiro contato com pacientes. Tal mudança poderia submeter o estudante a sentimentos de ansiedade, insegurança e culpa, sobretudo quando se depara com sua inabilidade inicial diante das novas exigências.

No mesmo sentido, o trabalho de Feijó DH, et al. (2018) indica que o terceiro e quarto anos de graduação podem piorar a autoestima do aluno devido ao início das cobranças pelos pacientes e professores com relação aos conhecimentos já adquiridos, além de um aumento da cobrança familiar e pessoal. Ocorre também um maior acúmulo de disciplinas cursadas e o aluno começaria a lidar com conflitos éticos, dor e sofrimento humano.

Com relação à comparação entre a saúde mental do estudante no início e no fim do semestre, o estudo de Ferreira CMG, et al. (2016) indicou diferenças consideráveis entre os dois períodos. A prevalência de transtornos mentais comuns para o total de alunos no início do semestre foi de $35,8 \%$ e no final do semestre aumentou para $51,5 \%$. Em resposta ao formulário da pesquisa, os maiores percentuais de respostas afirmativas foram para a questão sobre se sentir nervoso, tenso ou preocupado, que passou de $71,6 \%$ no início do semestre para $78,4 \%$ no final do semestre.

Em seguida, no estudo de Ferreira CMG, et al. (2016), encontram-se as respostas sobre ficar cansado com facilidade, que passou de $58,6 \%$ para $65,7 \%$, se sentir cansado o tempo todo, de $44,0 \%$ para $55,2 \%$, e dormir mal, de $35,1 \%$ para $51,5 \%$ no início e final do semestre, respectivamente. Tais resultados poderiam ser explicados pelo acúmulo de avaliações, trabalhos, cobranças e pela privação do sono, comuns na etapa final do semestre. Esse estudo indicou que a qualidade ruim do sono foi um fator associado à presença dos transtornos mentais comuns, com chance 3,3 vezes maior de um possível caso de transtornos mentais comuns.

A privação do sono foi um aspecto também abordado em Medeiros MRB, et al. (2018), que afirma que muitos estudantes de medicina têm perdido a qualidade do sono. Devido à grande exigência de conteúdo e carga horária, é prática comum entre os estudantes trocar noites de descanso por horas de estudo, especialmente em períodos de provas. Além disso, o sono irregular dificulta os processos mentais de pensamento e julgamento, o que resulta em dificuldade de concentração e de tomada de decisões (AQUINO DR, et al., 2019).

Na pesquisa de Leão AM, et al. (2018) sobre a prevalência e fatores associados à depressão e ansiedade entre estudantes universitários da área da saúde em uma grande cidade do nordeste foi concluído que um dos aspectos mais fortemente associados à ansiedade foi a baixa quantidade e qualidade de sono, sendo a insônia uma das principais características referidas pelos alunos avaliados. Assim, torna-se claro que a quantidade inadequada de sono pode diminuir o rendimento das atividades cotidianas e pode acarretar em outros problemas de saúde como ansiedade. 
Outra realidade encontrada nos estudos vivida por estudantes de medicina é a do esgotamento físico e mental, manifestada em última instância pela Síndrome de Burnout. O estudo de Medeiros MRB, et al. (2018) mostrou que um grande número de estudantes apresentou, em conjunto, níveis moderados e altos de exaustão emocional e de despersonalização, essa última entendida como o distanciamento emocional do indivíduo em relação a colegas e pacientes. O estudo concluiu ainda que caso os fatores desencadeantes do esgotamento profissional se mantivessem, seria possível que os acadêmicos desenvolvessem Síndrome de Burnout. A falta de tempo livre, mais uma vez devido à alta exigência do curso, foi apontada também como um fator que exerce grande impacto na piora da qualidade de vida e das relações sociais desse grupo.

Por fim, um último fator de grande influência são as preocupações financeiras decorrentes da impossibilidade de trabalhar devido ao período integral do curso médico. No estudo de Aquino DR, et al. (2019) sobre sintomas de depressão em universitários de medicina, a maioria dos universitários que participaram da pesquisa informou nunca ter trabalhado. A dependência financeira de terceiros por um longo período, especialmente entre os alunos de baixa renda, pode gerar perda da qualidade de vida e quadros de angústia e depressão.

Em conclusão, os diversos aspectos relacionados à extensão da carga horária e do nível de exigência do curso influenciam diretamente a qualidade de vida e a saúde mental dos estudantes de medicina, com consequências nos mais diversos âmbitos de suas vidas- (AQUINO DR, et al., 2019).

\section{Aprendizagem baseada em problemas e saúde mental}

Outro ponto também associado a esse contexto é o uso da metodologia baseada em problemas em algumas universidades. O estudo de Rizwan F, et al. (2016), conduzido de maio a setembro de 2014, dividiu estudantes de uma mesma faculdade de medicina em dois grupos: um grupo foi submetido à metodologia tradicional, enquanto o outro participou da metodologia de ensino baseado em problemas. Após a realização de um teste ao final da pesquisa, os resultados mostraram melhor desempenho pelos alunos que foram direcionados à coorte da metodologia não tradicional. Dessa maneira, essa pesquisa corrobora a ideia de que novos métodos de ensino possivelmente continuarão ganhando espaço nas escolas médicas.

Além da eficácia de aprendizagem, a metodologia baseada em problemas tem sido considerada positiva na interação social dos estudantes com os colegas. Esse tipo de atividade estimula os alunos a pensarem nos casos clínicos em questão e a discutirem o que outras pessoas do grupo pensam a respeito. Dessa maneira, é criada uma atmosfera propícia à interação social. Ademais, é comum que os alunos se sintam mais motivados a contribuírem com suas ideias nesse tipo de metodologia (WHITE HB e USHER DC, 2015).

Brigati JF, et al. (2020) abordam possíveis situações de ansiedade que podem ser geradas pela metodologia ativa de ensino. A necessidade de participação do estudante na aprendizagem baseada em problemas pode causar insegurança. Isso não é necessariamente negativo, uma vez que é uma maneira de estimular o aluno a estudar o material previamente às aulas e se preparar melhor. Não obstante, o excesso de estresse pode ser prejudicial. Por essa razão, muitos estudantes desenvolveram mecanismos de enfrentamento para lidarem melhor com o estresse. Os mecanismos mais prevalentes na pesquisa foram: busca de informações; solução de problemas; autoconfiança e busca por ajuda. Apesar de a real eficácia destes mecanismos não estar confirmada, isto pode ser considerado o alicerce para a elaboração de potenciais intervenções para a ansiedade nas salas de aula.

\section{CONSIDERAÇÕES FINAIS}

Os fatores envolvidos na formação médica influenciam na qualidade de vida e saúde mental dos estudantes. Conhecer a realidade brasileira é importante para estabelecer medidas a serem implementadas e criar mecanismos de suporte para esse grupo. Entender como esse contexto implica no adoecimento dos estudantes e as consequências da permanência desse cenário, como o aumento do consumo de drogas e álcool, maior incidência de casos de ansiedade, depressão e suicídio, permitirá estabelecer uma formação médica com maior qualidade. É necessário identificar as necessidades dos estudantes e buscar alternativas que possam acolher esse público, direcionando esse olhar de forma mais ampla, observando suas características sociais, culturais, os aspectos emocionais e físicos. 


\section{REFERÊNCIAS}

1. AMORIM BB, et al. Saúde Mental do Estudante de Medicina: Psicopatologia, Estresse, Sono e Qualidade de Vida. Revista Psicologia, Diversidade e Saúde, 2018; 7(2): 245-254.

2. AQUINO DR, et al. Sintomas de depressão em universitários de medicina. Boletim Academia Paulista de Psicologia, 2019; 39(96): 81-95.

3. ARINO DO, BARDAGI MP. Relação entre Fatores Acadêmicos e a Saúde Mental de Estudantes Universitários. Psicologia em pesquisa, 2018; 12(3): 44-52.

4. BALDASSIN S, et al. The characteristics of depressive symptoms in medical students during medical education and training: a cross-sectional study. BMC medical education, 2008; 8: 60.

5. BARBOSA ML, et al. Burnout Prevalence and Associated Factors Among Brazilian Medical Students. Clinical Practice \& Epidemiology in Mental Health, 2018; 14: 188-195.

6. BORGES GBM, et al. Defense mechanisms and quality of life of medical students according to graduation phase. Trends Psychiatry Psychother, 2020; 42(1): 74-81.

7. BRENNEISEN MF, et al. Factors associated to depression and anxiety in medical students: a multicenter study. BMC medical education, 2016; 16(1): 282.

8. BRIGATI JR, et al. How do undergraduates cope with anxiety resulting from active learning practices in introductory biology?. Plos One, 2020; 15(8).

9. CASTALDELLI-MAIA JM, et al. Stressors, psychological distress, and mental health problems amongst Brazilian medical students. International Review of Psychiatry, 2019; 31(8): 603-607.

10. CHAGAS NB, et al. Qualidade de Vida de Estudantes de Medicina em um Curso que Adota Metodologias Ativas de Ensino-Aprendizagem. Revista Brasileira de Educação Médica, 2018; 42(4): 96-102.

11. CUNHA DHF, et al. Percepção da qualidade de vida e fatores associados aos escores de qualidade de vida de alunos de uma escola de medicina. Jornal Brasileiro de Psiquiatria, 2017; 66(4).

12. FEIJÓ DH, et al. Dilemas e frustrações do estudante de medicina: estudo transversal. Pará Research Medical Journal, 2018; 1(3).

13. FERREIRA CMG, et al. Prevalência de Transtornos Mentais Comuns e Fatores Associados em Estudantes de Medicina: um Estudo Comparativo. Revista Brasileira de Educação Médica, 2016; 40(2): 268-277.

14. FONTANA MCP, et al. Burnout syndrome, extracurricular activities and social support among Brazilian internship medical students: a cross-sectional analysis. BMC medical education, 2020; 20(1): 81.

15. GHODASARA SL, et al. Assessing student mental health at the Vanderbilt University School of Medicine. Academic Medicine, 2011; 86(1): 116-21.

16. $\mathrm{KOCH} \mathrm{J}$, et al. "Sage on the stage or guide on the side"-Undergraduate nursing students' experiences and expectations of bioscience tutors in a blended learning curriculum: A qualitative study. Journal of Clinical Nursing, 2020; 29(6): 863-871.

17. LEAO AM, et al. Prevalência e Fatores Associados à Depressão e Ansiedade entre Estudantes Universitários da Área da Saúde de um Grande Centro Urbano do Nordeste do Brasil. Revista Brasileira de Educação Médica, 2018; 42(4): 55-65.

18. MAYER FB, et al. Factors associated to depression and anxiety in medical students: a multicenter study. BMC Medical Education, 2016;16: 282.

19. MEDEIROS MRB, et al. Mental health of first-year academics in the medical school: a gender approach. Revista Brasileira de Educação Médica, 2018; 42(3): 214-221.

20. MORGAN HL, et al. Consumo de Estimulantes Cerebrais por Estudantes de Medicina de uma Universidade do Extremo Sul do Brasil: Prevalência, Motivação e Efeitos Percebidos. Revista Brasileira de Educação Médica, 2017; 41(1): 102-109.

21. MOUTINHO ILD, et al. Mental health and quality of life of Brazilian medical students: Incidence, prevalence, and associated factors within two years of follow-up. Psychiatry Research, 2019; 306-312.

22. MOUTINHO ILD. Estresse, ansiedade, depressão, qualidade de vida e uso de drogas ao longo da graduação em medicina: estudo longitudinal (Doutorado - Programa de Pós-Graduação em Saúde) - Universidade Federal de Juiz de Fora, Juiz de Fora, 2018, 151 p.

23. PACHECO JP, et al. Mental health problems among medical students in Brazil: a systematic review and meta-analysis. Associação Brasileira de Psiquiatria, 2017; 39(4): 369-378.

24. PAGNIN D, DE QUEIROZ V. Comparison of quality of life between medical students and young general populations. Education for health (Abingdon, England), 2015; 28(3): 209-12.

25. PURIM KSM, et al. Privação do sono e sonolência excessiva em médicos residentes e estudantes de medicina. Revista do Colégio Brasileiro de Cirurgiões, 2016; 43(6): 438-444.

26. PUTHRAN R, et al. Prevalence of depression amongst medical students: a meta-analysis. Medical Education, 2016; 50(4): 456-68.

27. RIZWAN F, et al. Aprendizagem baseada em problemas em comparação com a aprendizagem baseada em palestras entre estudantes de medicina. Journal Of Pakistan Medical Association, 2016; 66.

28. VASCONCELOS TC. Prevalência de Sintomas de Ansiedade e Depressão em Estudantes de Medicina. Revista Brasileira de Educação Médica, 2015; 39(1): 135-142.

29. WHITE HB, USHER DC. Engaging novice researchers in the process and culture of science using a "Pass-the-problem" case strategy. Biochem Mol Biol Educ., 2015; 43(5): 341-4. 